\title{
Componentes da produtividade da água modelados por sensoriamento remoto em limoeiros irrigados de Minas Gerais
}

\author{
Antônio Heriberto de Castro Teixeira ${ }^{1(*)}$, João Batista Ribeiro da Silva Reis ${ }^{2}$, Janice Freitas Leivas ${ }^{3}$, \\ Gustavo Bayma Siqueira da Silva ${ }^{4}$, Tiago Barbosa Struiving ${ }^{5}$ \\ ${ }^{1}$ Pesquisador, Embrapa Monitoramento por Satélite, Campinas, São Paulo, heriberto.teixeira@embrapa.br \\ ${ }^{2}$ Pesquisador, Empresa de Pesquisa Agropecuária de Minas Gerais, Nova Porteirinha, Minas Gerais, jbrsreis@epamig.br \\ ${ }^{3}$ Pesquisadora, Embrapa Monitoramento por Satélite, Campinas, São Paulo, janice.leivas@embrapa.br \\ ${ }^{4}$ Analista, Embrapa Monitoramento por Satélite, Campinas, São Paulo, gustavo.bayma@embrapa.br \\ ${ }^{5}$ Agrônomo, Associação dos Produtores de Limão, Matias Cardoso, Minas Gerais, struiving@gmail.com \\ ${ }^{(*)}$ Autor para correspondência
}

INFORMAÇÕES

História do artigo:

Recebido em 16 de Junho de 2017

Aceito em 10 de agosto de 2017

Termos para indexação:

SAFER

manejo de irrigação

Citrus limon $\mathrm{L}$

\section{RESUMO}

Objetivando subsidiar o manejo racional da cultura do limoeiro irrigado sob diferentes sistemas de irrigação e porta-enxertos, imagens Landsat 8 (L8) e dados climáticos foram usadas através do algoritmo SAFER, ao longo do ano de 2015, para a modelagem dos componentes da produtividade da água da cultura, no município de Matias Cardoso, Estado do Minas Gerais, Sudeste do Brasil. Para os sistemas de irrigação localizada, micro aspersão e gotejamento, o período de maior consumo hídrico, com a evapotranspiração real (ET) acima de 4,0 $\mathrm{mm} \mathrm{dia}^{-1}$, foi da segunda quinzena de fevereiro à primeira quinzena de março, enquanto que para o sistema de irrigação por pivô, os máximos, com ET acima de $4,5 \mathrm{~mm} \mathrm{dia}^{-1}$ aconteceram do final de outubro para o início de novembro. Considerando-se todas os sistemas de irrigação e porta-enxertos utilizados, a produtividade da água baseada na ET ( $\mathrm{PA}_{\mathrm{ET}}$ ) média foi de $2,4 \mathrm{~kg} \mathrm{~m}^{-3}$. Os valores de coeficiente de cultua $\left(\mathrm{K}_{\mathrm{c}}\right)$ variaram de acordo com as fases fenológicas consideradas na região de estudo, sendo de 1,01 e 0,97 para as duas floradas; de 1,04 e 0,80 nas duas fases de crescimento dos frutos; e de 0,88, 1,06 e de 0,72 nos três picos de colheita. Os resultados indicaram a possibilidade de economia de água no manejo de irrigação com manutenção da produtividade de limões.

(c) 2017 SBAgro. Todos os direitos reservados.

\section{Introdução}

O Brasil é o maior produtor mundial de citros, sendo que o estado de Minas Gerais (MG) ostenta a segunda colocação no ranking nacional na produção do limão (Citrus limon L.), cultura que vem se destacando nas condições semiáridas do Norte do Estado, em virtude do projeto de irrigação do Jaíba. o projeto foi criado com o objetivo de promover o desenvolvimento regional integrado com base na agricultura irrigada, agroindústria e agropecuária. Mais da metade da área do perímetro é destinada à fruticultura, com destaque para a produção de limão.

O município de Jaíba responde por 39,5\% da produção mineira de limão, e Matias Cardoso, por 31,3\%. o projeto Jaíba está localizado nesses dois municípios, que respondem por cerca de $70 \%$ da produção estadual, com grande 
participação no mercado externo. Os investimentos nos tratos culturais, nas certificações e na qualidade dos frutos estão trazendo bons resultados para os produtores credenciados na Associação dos Produtores de Limão do Jaíba (ASLIM).

Com as condições climáticas favoráveis para irrigação, a fruticultura na região semiárida do Norte de Minas, vêm se expandindo, substituindo a vegetação natural rapidamente. Sendo o limoeiro irrigado uma das principais fruteiras, análises dos componentes da produtividade da água são importantes para o manejo racional da água, nestas condições. Para estas análises, torna-se importante, além da obtenção dos dados de produtividade, quantificações da evapotranspiração (TEIXEIRA; LEIVAS, 2017).

Para quantificação dos componentes da produtividade da água, distinções são feitas entre as evapotranspirações de referência (ET $)$ e real (ET). A ET 0 é considerada como o fluxo hídrico de uma superfície de referência como a grama com características específicas, enquanto a ET é o fluxo hídrico real que ocorre envolvendo todas as condições ambientais. Por um lado, em culturas bem irrigadas, os valores do indicador razão da evapotranspiração - $\mathrm{ET}_{\mathrm{r}}\left(\mathrm{ET} / \mathrm{ET}_{0}\right)$, conhecido como coeficiente de cultura $\left(\mathrm{K}_{\mathrm{c}}\right)$, é usado para estimativa dos requerimentos hídricos (ALLEN et al., 1998; CONSOLI; PAPA, 2013). Por outro lado, em vegetação natural, este indicador pode ser usado para caracterizar as condições de estresse hídrico (LU et al., 2011).

Poucas pesquisas já foram realizadas com relação aos componentes da produtividade da água com a cultura do limoeiro irrigado no Brasil, e, quando acontecem, são geralmente baseadas em experimentos de campo (JUNIOR et al. 2008), acarretando em valores pontuais específicos. Além das variações espaciais destes componentes de acordo com as condições de umidade do solo, as quais dependem do manejo de irrigação, estes componentes podem ainda variar com o porta-enxerto utilizado (GARCIA-TEJERO et al., 2010; PEDROSO et al., 2014; ROBLES, J.M.; BOTÍA, P.; PÉREZ-PÉREZ, J.G., 2017), especialmente nas condições semiáridas.

A redução da quantidade de água aplicada via irrigação nas condições semiáridas sem afetar a produção é desejada, principalmente nas situações de competição hídrica da agricultura com outros setores nas regiões semiáridas. Germaná e Sardo (2004) reportaram um aumento no peso dos frutos e economia de água aplicada na irrigação através com déficits hídricos em determinados estágios fenoo lógicos da cultura da laranja.

Apesar da obtenção dos componentes da produtividade da água do limoeiro poder ser realizada através de medições do balanço hídrico em campo (JUNIOR et al. 2008), destaca-se a importância da utilização do sensoriamento remoto por imagens de satélites para tal finalidade, pela vantagem de obtenção dos valores médios destes componentes abrangendo diferentes condições hídricas e agros-ecossistemas (KAMBLE et al., 2013; TEIXEIRA; LEIVAS, 2017).

O objetivo do atual trabalho foi a aplicação do algoritmo SAFER - Simple Algorithm for Evapotranspiration Retrieving (TEIXEIRA; LEIVAS; SILVA, 2016), com imagens Landsat 8 (L8) em conjunto com dados climáticos, cobrindo as fases fenológicas do limoeiro cultivado sob diferentes métodos de irrigação e porta-enxertos no ano de 2015, objetivando a quantificação dos componentes da produtividade da água nas condições semiáridas do Norte de Minas Gerais. Os resultados podem subsidiar critérios para decisões gerenciais no manejo de irrigação da cultura, no Sudeste do Brasil, considerando-se as condições de escassez hídrica natural, de mudanças de uso da terra e competição por água entre os diferentes setores.

\section{Material e métodos}

\section{Área de estudo e dados utilizados}

A Figura 1 mostra a localização das áreas cultivadas com limoeiro cultivado sob diferentes sistemas de irrigação nas fazendas Esperança (ES), Yamada (YA), Santa Fé (SF), Saara (SA), Marazul (MA) e Tropical (TR), situadas no município de Matias Cardoso, estado do Minas Gerais (MG), Sudeste do Brasil, juntamente com a estação agrometeorológica. As cenas L8 usadas na modelagem foram de ponto/ órbita 218/70, 218/71 e 219/70, com sobreposições das passagens do satélite na área estudada. Uma composição RGB ("Red", "Green", "Blue") para o dia juliano (DJ) 216 é usada como base.

O Norte de Minas Gerais apresenta clima tropical, sendo caracterizado por uma grande diversidade física, social e econômica, com clima semiárido, temperaturas elevadas, chuvas concentradas em poucos meses do ano. Apresenta diversidade de formações vegetais típicas, onde boa parcela que corresponde ao Bioma Caatinga entra em contato ecossistêmico com o Cerrado (COSTA; RUAS; PEREIRA, 2010). Devido ao desenvolvimento tecnológico da irrigação nas vizinhanças do Rio São Francisco, a vegetação natural vem rapidamente sendo substituída por culturas irrigadas, principalmente fruteiras.

No perímetro irrigado Jaíba a produtividade média do limoeiro é de $25 \mathrm{t} \mathrm{ha} \mathrm{a}^{-1}$. As colheitas ocorrem durante o ano inteiro, com dois períodos de pico - entre novembro/janeiro e junho/julho. A Tabela 1 apresenta as fases fenológicas generalizadas consideradas pelos produtores de limão no Norte de Minas Gerais.

Na região do Norte de Minas, devido à proximidade do equador, o limoeiro irrigado tem múltiplas floradas durante o ano, com as parcelas irrigadas apresentando diferentes fases em uma mesma planta. Contudo a intensidade de 


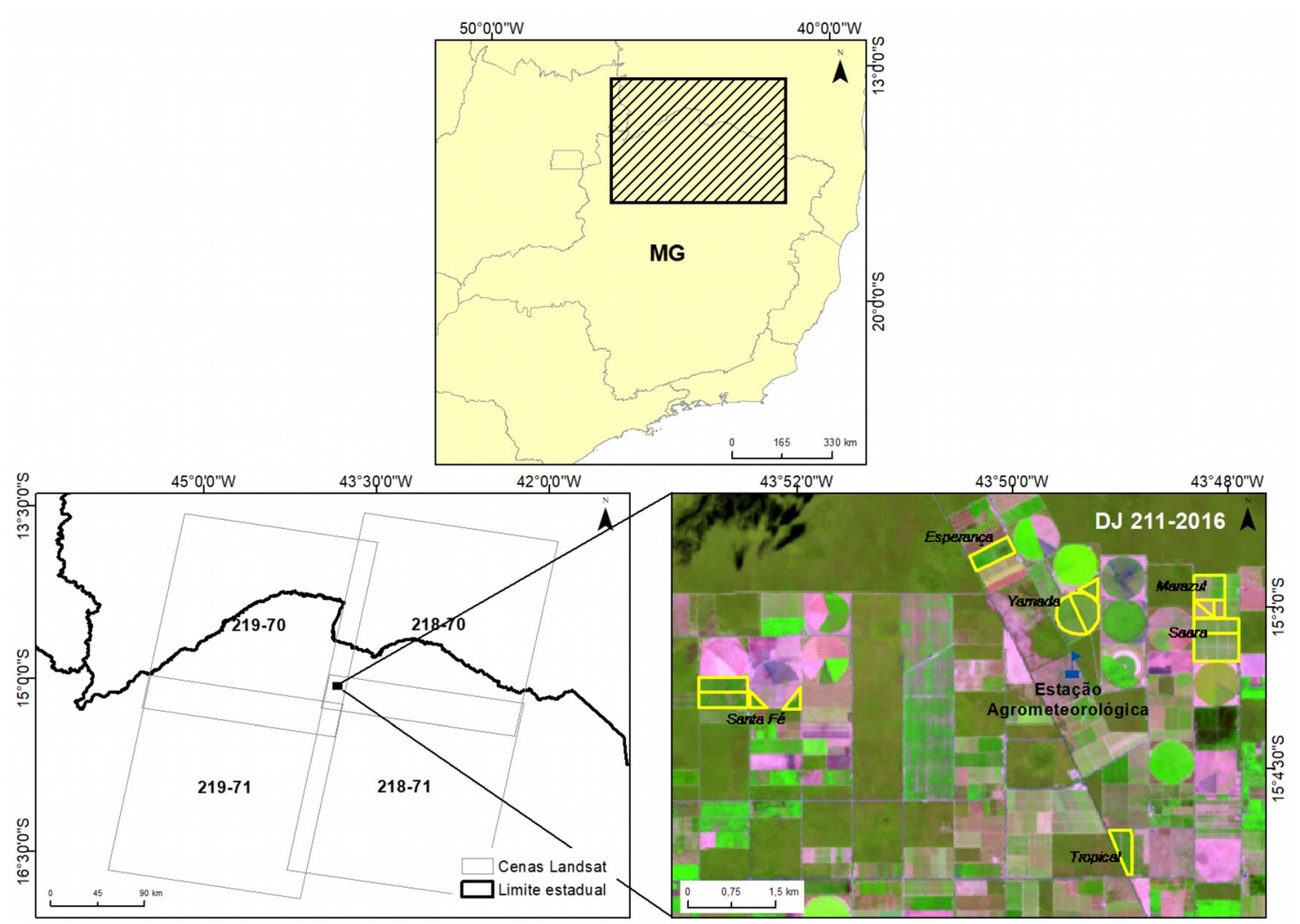

Figura 1. Localização das áreas cultivadas com a cultura do limoeiro irrigado nas fazendas Esperança (ES), Yamada (YA), Santa Fé (SF), Saara (SA), Marazul (MA) e Tropical (TR), localizadas no município de Matias Cardoso, estado do Minas Gerais (MG), Sudeste do Brasil, juntamente com a estação agrometeorológica e as cenas 218/70, 218/71 e 219/70 do Landsat 8. Uma composição RGB ("Red”, "Green”, "Blue") para o dia juliano (DJ) 216 é usada como base.

Tabela 1. Fases fenológicas consideradas para a cultura do limoeiro irrigado no Norte de Minas Gerais.

\begin{tabular}{|c|l|l|l|l|l|l|l|l|l|l|l|l|}
\hline $\begin{array}{l}\text { MÊS/ } \\
\text { FASE }\end{array}$ & $\begin{array}{l}\text { JA } \\
\text { N }\end{array}$ & $\begin{array}{l}\text { FE } \\
\text { V }\end{array}$ & $\begin{array}{l}\text { MA } \\
\text { R }\end{array}$ & $\begin{array}{l}\text { AB } \\
\text { R }\end{array}$ & $\begin{array}{l}\text { MA } \\
\text { I }\end{array}$ & $\begin{array}{l}\text { JU } \\
\text { N }\end{array}$ & $\begin{array}{l}\text { JU } \\
\text { L }\end{array}$ & $\begin{array}{l}\text { AG } \\
\text { O }\end{array}$ & $\begin{array}{l}\text { SE } \\
\text { T }\end{array}$ & $\begin{array}{l}\text { OU } \\
\text { T }\end{array}$ & $\begin{array}{l}\text { NO } \\
\text { V }\end{array}$ & $\begin{array}{l}\text { DE } \\
\text { Z }\end{array}$ \\
\hline F & & & $\mathrm{X}$ & $\mathrm{X}$ & & & & $\mathrm{X}$ & $\mathrm{X}$ & & & \\
\hline CF & & & & $\mathrm{X}$ & $\mathrm{X}$ & $\mathrm{X}$ & & & $\mathrm{X}$ & $\mathrm{X}$ & $\mathrm{X}$ & \\
\hline PC & $\mathrm{X}$ & $\mathrm{X}$ & & & & $\mathrm{X}$ & $\mathrm{X}$ & $\mathrm{X}$ & & & $\mathrm{X}$ & $\mathrm{X}$ \\
\hline
\end{tabular}

*F - Florada; CF - Crescimento dos frutos; PC - Pico de colheita

florescimento depende das condições climáticas ao longo dos anos.

A Tabela 2 apresenta as diferentes variedades analisadas no ano de 2015, com respectivos porta-enxertos, produtividade (P), área cultivada (A) e data de plantio, sob os sistemas de irrigação (SI) microaspersão (M), gotejamento (G) e pivô (P).

Seguindo a Tabela 2, as análises envolveram plantas de 6 a 20 anos de idade com espaçamento de $7 \mathrm{~m}$ entre linhas e 5 m entre plantas, totalizando áreas de 122; 60,9 e 46,3 ha irrigados por microaspersão (M), gotejamento $(G)$ e pivô (P), respectivamente. A produtividade (P) variou de 14,2 a $37,5 \mathrm{tha}^{-1}$ ambos os limites, abaixo e acima da média da região, acontecendo para o sistema de irrigação (SI) M, mas com os porta-enxertos Fly Dragon e Cravo, nas fazendas Saara (SA) e Santa Fé (SF), respectivamente.
Uma estação agrometeorológica foi instalada na vizinhança das fazendas estudadas (ver Figura 1), a qual foi usada em conjunto com as imagens L8 adquiridas em diferentes condições termo hídricas do ano de 2015. Os dados de radiação solar global $\left(\mathrm{R}_{G}\right)$, temperatura do ar $\left(\mathrm{T}_{\mathrm{a}}\right)$ e evapotranspiração de referência ( $\mathrm{ET}_{0}$ ) foram usados em conjunto com os parâmetros obtidos por sensoriamento remoto, o albedo da superfície $\left(\alpha_{0}\right)$ e o índice da diferença de vegetação normalizado (NDVI) na estimativa da razão $\mathrm{ET} / \mathrm{ET}_{0}$ com aplicação do SAFER.

As sobreposições de imagens apresentadas na Figura 1 deu a oportunidade de análises dos componentes da produtividade da água do limoeiro envolvendo várias condições termo hídricas e de cobertura do solo pela cultura com diferentes métodos de irrigação e porta-enxertos. Um total de 27 imagens Landsat 8 foram adquiridas, sendo que 
Tabela 2. Variedades de limoeiro analisadas, com respectivos porta-enxertos, produtividade (P), área cultivada (A) e data de plantio, sob os sistemas de irrigação (SI) microaspersão (M), gotejamento (G) e pivô (P), no ano de 2015.

\begin{tabular}{|c|c|c|c|c|c|c|}
\hline Fazenda & $\begin{array}{c}\text { Porta } \\
\text { Enxerto }\end{array}$ & Copa & 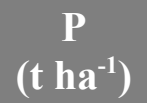 & $\begin{array}{c}\text { A } \\
\text { (ha) }\end{array}$ & Plantio & SI \\
\hline Esperança & Cravo & IAC5 & 26,2 & 22,00 & $03 / 2008$ & $\mathrm{M}$ \\
\hline \multirow{3}{*}{ Yamada } & Cravo & IAC5 & 30,5 & 10,93 & $01 / 2010$ & M \\
\hline & Citrumelo & Quebra Galho & 36,2 & 23,87 & $01 / 2010$ & $\mathrm{P}$ \\
\hline & Cravo & Quebra Galho & 27,7 & 22,41 & $01 / 2010$ & $\mathrm{P}$ \\
\hline \multirow{4}{*}{ Santa Fé } & Cravo & IAC5 & 37,5 & 21,66 & $09 / 2009$ & $\mathrm{M}$ \\
\hline & Cravo & IAC5 & 30,5 & 20,89 & $09 / 2009$ & $\mathrm{M}$ \\
\hline & Cravo & IAC5 & 16,2 & 3,06 & $03 / 2010$ & $\mathrm{G}$ \\
\hline & Cravo & IAC5 & 14,3 & 3,87 & $03 / 2010$ & $\mathrm{G}$ \\
\hline \multirow{2}{*}{ Saara } & Cravo & Quebra Galho & 34,1 & 35,78 & $08 / 2010$ & $\mathrm{G}$ \\
\hline & Fly Dragon & IAC5 & 40,1 & 18,15 & $08 / 2010$ & G \\
\hline \multirow{3}{*}{ Marazul } & Fly Dragon & IAC5 & 14,2 & 9,96 & $01 / 2012$ & M \\
\hline & Cravo & Quebra Galho & 24,8 & 5,48 & $01 / 2010$ & $\mathrm{M}$ \\
\hline & Cravo & IAC5 & 35,9 & 13,24 & $01 / 2007$ & $\mathrm{M}$ \\
\hline Tropical & Cravo & IAC5 & 21,2 & 17,85 & $11 / 2009$ & M \\
\hline
\end{tabular}

quando houve problemas de nebulosidade, os valores espaciais de $\alpha_{0}$ e NDVI foram interpolados sucessivamente, mas, na modelagem, utilizando-se os dados climáticos para os dias com céu encoberto, resultando em um total de 52 imagens ao longo do ano.

\section{Modelagem dos componentes da produtividade da água}

As bandas 1 a 7 foram usadas na determinação de $\alpha_{0} \mathrm{e}$ NDVI, enquanto que a temperatura da superfície $\left(T_{0}\right)$ foi obtida como resíduo no balanço de radiação. Detalhes da metodologia sçao descritos em Teixeira, Leivas, e SILVA (2016) e Teixeira e Leivas (2017), sendo aqui as equações principais resumidas. A opção de estimativa da $\mathrm{T}_{0}$ sem a região termal do satélite (método residual) deve-se a obtenção de uma melhor resolução espacial de $30 \mathrm{~m}$ para os componentes da produtividade da água compatível com a escala das parcelas irrigadas.

As radiações espectrais $\left(\mathrm{L}_{b}\right)$ foram computadas a partir dos números digitas (DN):

$$
\mathrm{L}_{\mathrm{b}}=\mathrm{aDN}+\mathrm{b}
$$

onde os coeficientes de regressão $a$ e $b$ foram obtidos nos meta arquivos.

O albedo planetário para cada banda do L8 $\left(\alpha_{\mathrm{pb}}\right)$ foi calculado como:

$$
\alpha p_{b}=\frac{L_{b} \pi d^{2}}{R_{T b} \cos \phi}
$$

onde $\mathrm{L}_{b}$ é a radiação espectral para os comprimentos de onda da banda $b\left(\mathrm{~W} \mathrm{~m}^{-2} \mathrm{sr}^{-1} \mu \mathrm{m}^{-1}\right)$, $\mathrm{d}$ é a distância relativa Terra-Sol; $\mathrm{R}_{\mathrm{Tb}}$ é a irradiância solar média no topo da atmosa fera para cada banda $\left(\mathrm{W} \mathrm{m}^{-2} \mu \mathrm{m}^{-1}\right)$ e $\square$ é o ângulo zenital.

$\mathrm{R}_{\mathrm{Tb}}$ para cada uma das bandas de 1 a 7 do sensor L8 foi calculada de acordo com a lei de Planck, integrando a radiação nos intervalos de comprimento de onda e considerando suas frações no espectro solar, assumindo o Sol com um corpo negro. Então para toda a faixa do visível o albedo planetário $\left(\alpha_{\mathrm{p}}\right)$ foi obtido como a soma total dos valores de $\alpha p_{b}$ para cada banda de acordo com seus pesos $\left(\mathrm{w}_{\mathrm{b}}\right)$.

$$
\alpha p=\sum w_{b} \alpha p_{b}
$$

Para a estimativa de $\alpha_{0}$, correções atmosféricas para os valores de $\alpha_{p}$ foram aplicadas através de regressões obtidas de medições conjuntas prévias de campo e por sensoriamento remoto.

A radiação atmosférica $\left(R_{a}\right)$ foi calculada através da lei de Stefan-Boltzmann:

$$
\mathrm{R}_{\mathrm{a}}=\sigma \varepsilon_{\mathrm{A}} \mathrm{T}_{\mathrm{a}}^{4}
$$

em que $\mathrm{T}_{\mathrm{a}}$ foi medida na estação agrometeorológica e a emissividade atmosférica $\left(\varepsilon_{\mathrm{A}}\right)$ foi calculada como:

$$
\varepsilon_{A}=a_{A}\left(\ln \tau_{s w}\right)^{b_{A}}
$$

onde $a_{A}$ e $b_{A}$ são os coeficientes de regressão.

Com a $R_{G}$ obtida na estação agrometeorológica e $\alpha_{0}$ por sensoriamento remoto, os valores diários da radiação solar 
refletida pela superfície cultivada $\left(R_{R}\right)$ foi estimada:

$$
\mathrm{R}_{\mathrm{R}}=\alpha_{0} \mathrm{R}_{\mathrm{G}}
$$

Os valores diários de $\mathrm{R}_{\mathrm{n}}$ foram calculados através da equação de Slob.

$$
R_{n}=\left(1-\alpha_{0}\right) R_{G}-a_{L} \tau_{s w}
$$

em que $\tau_{s w}$ é a transmissividade atmosférica que no atual trabalho foi considerada como a razão da $R_{G}$ para a radiação solar no topo da atmosfera e o coeficiente de regressão $a_{L}$ foi obtido espacialmente através da sua relação com $T_{a}$.

Tendo-se $R_{G}, R_{R}, R_{a}$ e $R_{n}$, a radiação de ondas longas emitida pela superfície cultivada $\left(\mathrm{R}_{\mathrm{s}}\right)$ foi obtida como resíduo no balanço de radiação:

$$
\mathrm{R}_{\mathrm{s}}=\mathrm{R}_{\mathrm{G}}-\mathrm{R}_{\mathrm{R}}+\mathrm{R}_{\mathrm{a}}-\mathrm{R}_{\mathrm{n}}
$$

Com a temperatura da superfície $\left(\mathrm{T}_{0}\right)$ obtida pela Eq. 4 , mas considerando $\mathrm{R}_{\mathrm{s}}$ e a emissividade da superfície $\left(\varepsilon_{\mathrm{s}}\right)$ como uma função do NDVI, a razão da $\mathrm{ET}$ para a $\mathrm{ET}_{0}$, indicador de umidade na zona das raízes, $\mathrm{ET}_{\mathrm{r}}$, foi modelada na escala de tempo diária:

$$
E T_{r}=\exp \left[a_{s f}+b_{s f}\left(\frac{T_{0}}{\alpha_{0} N D V I}\right)\right]
$$

onde $\mathrm{a}_{\mathrm{sf}} \mathrm{e} \mathrm{b}_{\mathrm{sf}}$ são os coeficientes de regressão.

Os valores da $\mathrm{ET}_{0}$, calculados com os dados da estação agrometeorológica pelo método de Penman-Monteith), foram multiplicados pelas imagens resultantes da Eq. 9, fornecendo os valores da ET pixel a pixel nas parcelas:

$$
E T=E T_{r} E T_{0}
$$

Para obtenção dos valores de $\mathrm{K}_{\mathrm{c}}$ as médias de $\mathrm{ET}_{\mathrm{r}}$ foram somados aos desvios padrões e os máximos para cada sistema de irrigação considerados para a garantia de ótimas condições de umidade do solo (ALLEN et al., 1998).

\section{Resultados e discussão}

\section{Condições climáticas}

A Figura 2 apresenta as tendências dos totais médios quinzenais dos pixels da precipitação ( $\mathrm{P}$ ) e da $\mathrm{ET}_{0}$ de acordo com intervalos de dias julianos (DJ) na área de estudo da Figura 1, no município de Matias Cardoso, Norte de Minas Gerais (MG), Sudeste do Brasil, durante o ano de 2015.

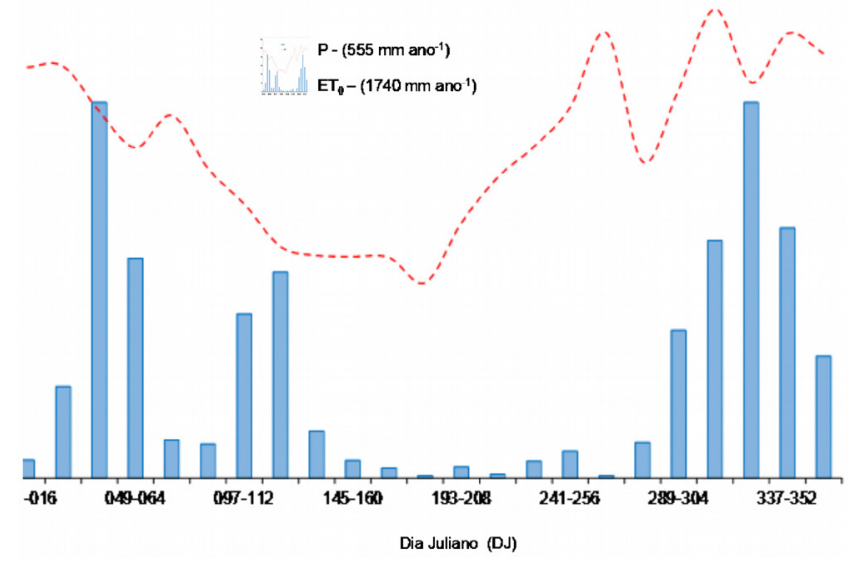

Figura 2. Tendências dos valores quinzenais da precipitação (P) e da evapotranspiração de referência (ETO) de acordo com intervalos de dias julianos (DJ) durante o ano de 2015 no município de Matias Cardoso, estado do Minas Gerais (MG), Sudeste do Brasil.

Devido às características semiáridas da região e a proximidade do equador, a precipitação (P) foi mais variável que a $\mathrm{ET}_{0}$. As chuvas se concentraram no início e no final do ano. Os períodos mais secos e longos, com $\mathrm{P}$ abaixo de 5 $\mathrm{mm}$, foram de DJ 160 a 289, inferiores a 10\% da ET . Entretanto percebe-se estiagens no início de janeiro e no período de DJ 064 a 097 durante a estação chuvosa, com P correspondente à apenas $4 \%$ da $\mathrm{ET}_{0}$. Esta carência de chuvas, aliada à elevados valores da demanda atmosférica (ET $)$, contribuiu com escassez hídrica natural para a cultura do limoeiro, demandando maior quantidade de irrigação suplementar.

\section{Umidade na zona das raízes}

A Figura 3 foi construída para detectar a sensibilidade de aplicação do SAFER com as imagens Landsat 8 nos agros-ecossistemas mistos da região semiárida, incluindo o limoeiro irrigado. Ela apresenta a distribuição espacial dos valores médios mensais do indicador $\mathrm{ET}_{\mathrm{r}}$, envolvendo diferentes condições termo hídricas do ano de 2015, na área de estudo no Norte do estado de Minas Gerais, Sudeste do Brasil. Destaques são dados para as áreas com a cultura do limoeiro sob irrigação por microaspersão (M), gotejamento (G) e pivô (P).

As variações espaciais e sazonais de $\mathrm{ET}_{\mathrm{r}}$ na área envolvendo culturas irrigadas e vegetação natural ao longo do ano são claras, confirmando a forte sensibilidade do SAFER. As diferenças espaciais são mais fortemente percebidas comparando-se as imagens do mês de janeiro, quando os valores de $\mathrm{ET}_{\mathrm{r}}$ ficam acima de 0,90 tanto para culturas irrigadas como para vegetação natural em grande parte da região, com aquela do mês de outubro, final do período mais seco, quando grande parte dos pixels apresentam valores com $\mathrm{ET}_{\mathrm{r}}$ igual a 0,00, representando as espécies de vegetação natural (ver Figura 2 e 3). Os valores maiores de 

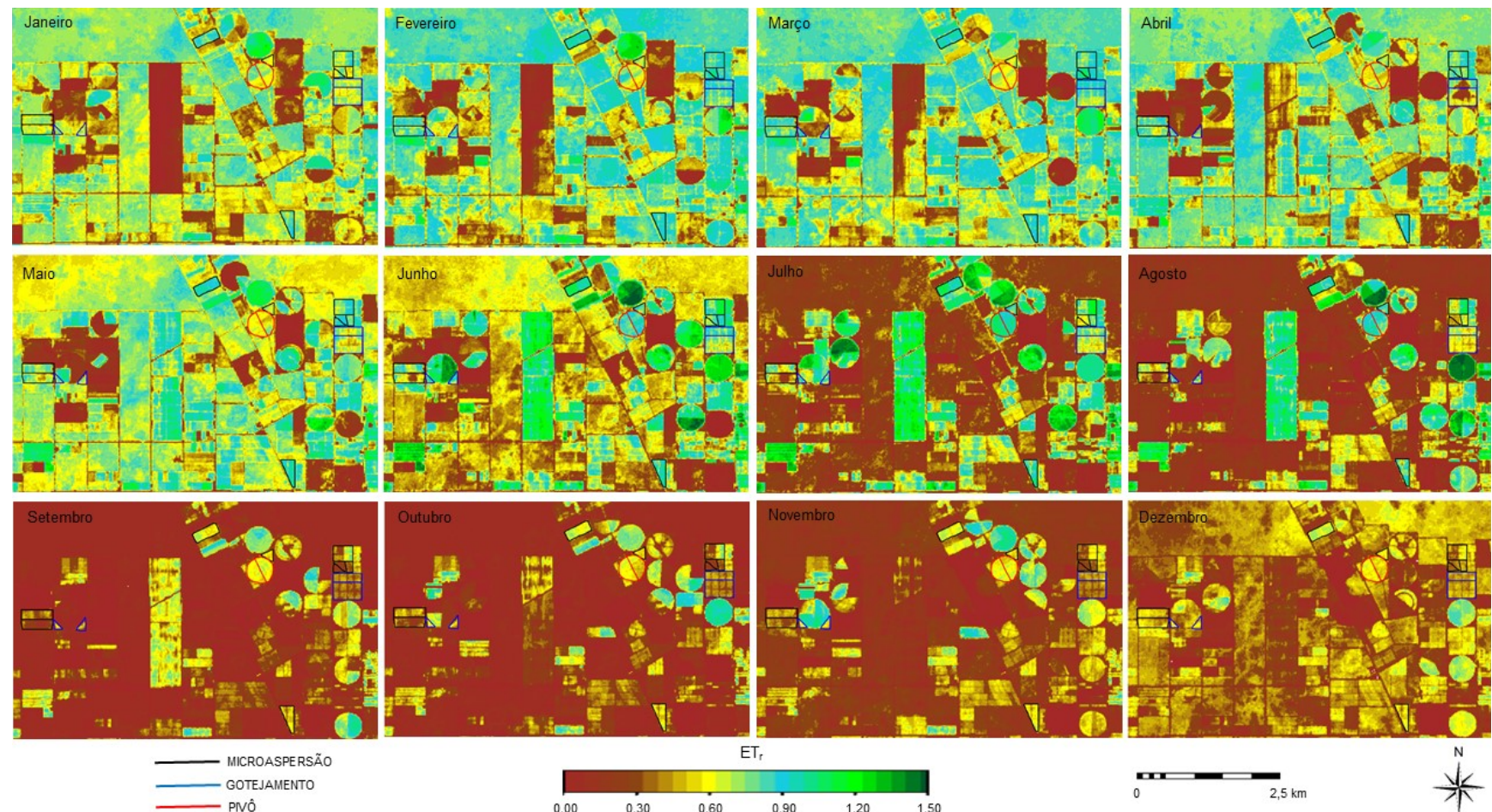

Figura 3. Distribuição espacial dos valores médios mensais do indicador $\mathrm{ET}_{r}$, para diferentes condições termo hídricas do ano de 2015 , na área de estudo no Norte de Minas Gerais. Destaques são dados para as áreas com a cultura do limoeiro sob irrigação por microaspersão (M), gotejamento $(\mathrm{G})$ e pivô $(\mathrm{P})$.

$\mathrm{ET}_{\mathrm{r}}$ no período mais seco, podem são atribuídos à irrigação. Dessa forma, em culturas agrícolas bem irrigadas, os valores máximos correspondem aos coeficientes de cultura, $\mathrm{K}_{\mathrm{c}}$ (ALLEN et al., 1998), enquanto os mínimos expressam condições de estresse hídrico (LU et al., 2011).

Zhang et al. (2012) reportaram valores de $\mathrm{ET}_{\mathrm{r}}$ em vegetações de desertos nas condições de clima temperado em Mongólia, China, variando de 0,16 a 0,75, similar a várias situações das espécies naturais do estudo corrente. Entretanto, Lu et al. (2011), na mesma região chinesa, encontraram valores acima de 1,00 para seis diferentes ecossistemas, equivalentes aos valores para as culturas bem irrigadas da Figura 3.

\section{Evapotranspiração real}

A Figura 4 mostra a distribuição espacial dos valores anuais da ET na área de estudo com destaque para as áreas cultivadas com limoeiro (Figura 4a) e as médias diárias extraídas dos pixels dentro das parcelas com a cultura (Figura 4b) sob os sistemas de irrigação por micro aspersão (M), gotejamento (G) e pivô (P), no ano de 2015, no município Matias Cardoso, Norte de Minas Gerais, Sudeste do Brasil.

De acordo com a Figura $4 \mathrm{a}$, considerando-se todos os agros-ecossistemas da área estudada, boa parte das culturas agrícolas bem irrigadas apresentaram valores anuais da ET entre 1000 a $2000 \mathrm{~mm} \mathrm{ano}^{-1}$. Entretanto percebem-se variações de acordo com o sistema de irrigação utilizado para a cultura do limoeiro, cujos valores estiveram entre 622 a $1573 \mathrm{~mm}^{-1} \mathrm{ano}^{-1}, 690$ a $1568 \mathrm{~mm}$ ano $^{-1}$ e 707 a $1689 \mathrm{~mm} \mathrm{ano}^{-1}$, respectivamente nos sistemas de irrigação por microaspersão (M), gotejamento (G) e pivô (P). Era de se esperar menores taxas para o sistema $\mathrm{G}$ que para $\mathrm{M}$, entretanto outros fatores relacionados aos manejos culturais estão envolvidos nos consumos hídricos, principalmente o emprego de porta-enxertos diferentes (GARCIA-TEJERO et al., 2010; PEDROSO et al., 2014; ROBLES, J.M.; BOTÍA, P.; PÉREZ-PÉREZ, J.G., 2017). Dos três sistemas de irrigação analisados, o sistema $\mathrm{P}$ foi o que apresentou os maiores valores de ET.

Analisando-se a tendência dos valores diários da ET ao longo do ano, embora o total anual para o sistema P tenha sido mais elevado que aqueles para irrigação localizada $\mathrm{M}$ e $G$, isto não aconteceu em todos os períodos ao longo do ano. $\mathrm{Na}$ estação chuvosa, de janeiro até junho os valores diários para o sistema $\mathrm{P}$ representou $88 \%$ daqueles para $\mathrm{M}$ e $G$, enquanto que para o período naturalmente mais seco de junho à primeira quinzena de novembro foi $30 \%$ superior, e a partir daí ficaram superiores em apenas $8 \%$ coincidindo com o retorno das precipitações (ver Figuras 2 e 4). Para os sistemas de irrigação localizada M e G, o período de maior consumo hídrico, com a ET acima de 4,0 $\mathrm{mm} \mathrm{dia}^{-1}$ foi da segunda quinzena de fevereiro à primeira quinzena de março, enquanto que para o sistema $\mathrm{P}$, os máximos, com ET acima de 4,5 $\mathrm{mm} \mathrm{dia}^{-1}$ aconteceram do final de outubro para o início de novembro.

Considerando todos os sistemas de irrigação, o 


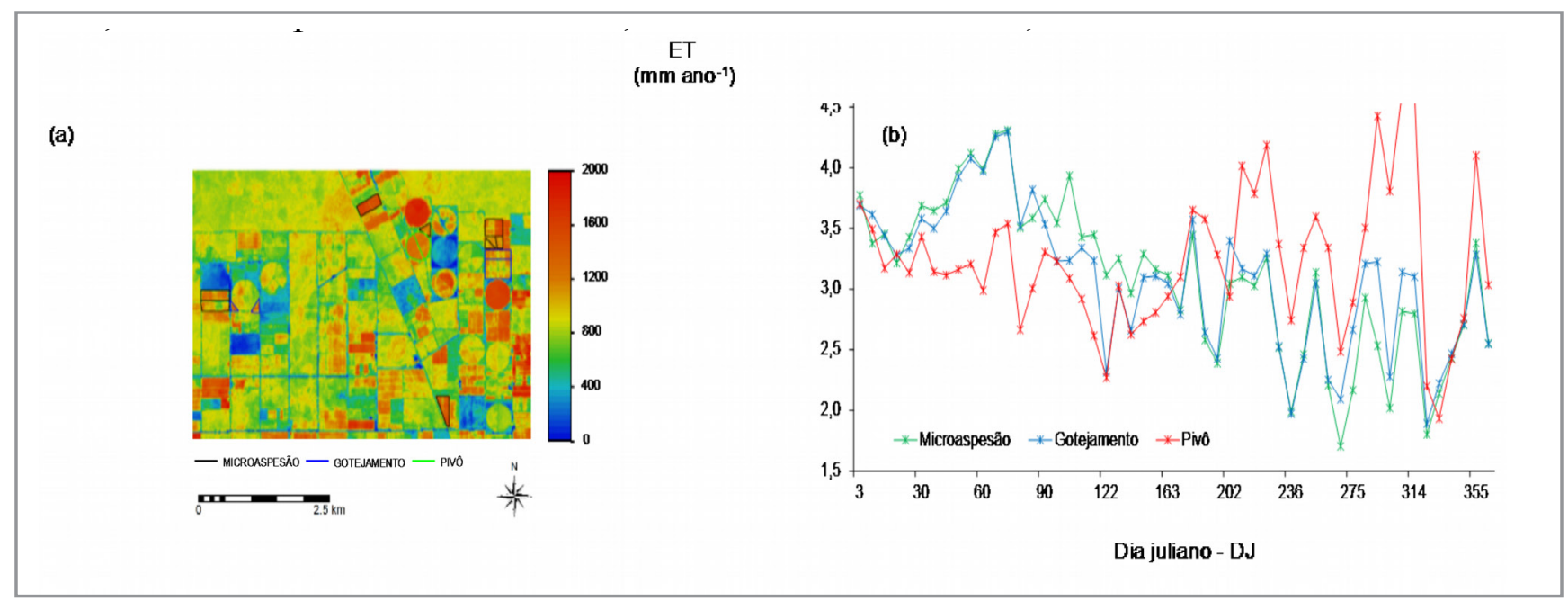

Figura 4. Variações espaciais e temporais da evapotranspiração real (ET) na área com limoeiros irrigados no ano de 2015 no Norte de Minas Gerais, com destaques para as parcelas cultivadas com os sistemas de irrigação por micro aspersão (M), gotejamento (G) e pivô (P). (a) distribuição espacial dos valores anuais; (b) tendências dos valores médios diários.

valor mínimo que ocorreram no final do ano foi de $1,7 \mathrm{~mm}$ dia ${ }^{-1}$ para micro aspersão $(M)$, enquanto que a média para todos os sistemas foi de $3,1 \mathrm{~mm} \mathrm{dia}^{-1}$. Consoli e Papa (2013) através de medições de campo do balanço de energia em pomar de laranja irrigado por gotejamento, encontraram faixas da ET entre 0,6 a 4,1 $\mathrm{mm}_{\text {dia }}^{-1}$, com média de 2,2 $\mathrm{mm}$ dia ${ }^{-1}$ nas condições semiáridas do Mediterrâneo. Yang, Yano e LI (2003) reportaram também o valor mínimo de 0,6 $\mathrm{mm} \mathrm{dia}^{-1}$, mas um máximo de $4,4 \mathrm{~mm}_{\text {dia }}{ }^{-1}$ em laranja sob condições de estufa. Embora com médias inferiores às do estudo corrente, os valores máximos foram bem próximos. Entretanto, Junior et al. (2008) reportaram uma faixa de ET muito próxima ao do estudo corrente, entre 1,7 e 4,3 $\mathrm{mm}$ dia $^{-1}$, resultantes de medições lisimétricas em limoeiro irrigado por gotejamento e porta-enxerto citrumelo, no Sudeste do Brasil, o que traz forte confiança nos resultados da aplicação do SAFER com imagens Landsat 8.

\section{Produtividade da água}

A Tabela 3 mostra os valores anuais da ET, da $\mathrm{ET}_{\mathrm{r}} \mathrm{e}$ da produtividade da água baseada na evapotranspiração $\left(\mathrm{PA}_{\mathrm{ET}}\right)$ para o limoeiro sob os diferentes sistemas de irrigação e porta-enxertos nas fazendas produtoras do município Matias Cardoso, Norte de Minas gerais, Sudeste do Brasil.

De acordo com a Tabela 3, para os sistemas de micro aspersão (M) e gotejamento (G), existem as situações de condução do limoeiro em que os porta enxertos Fly Dragon e Cravo são combinados com as variedades IAC5 e Quebra Galho, enquanto que para o pivô de irrigação (P) tem-se os porta-enxertos Cravo e Citrumelo ambos usados com a variedade Quebra Galho.

As taxas anuais mais elevadas da ET ocorreram na Fazenda ES com o porta-enxerto Cravo e a copa IAC5 sob o sistema de irrigação $\mathrm{M}$, enquanto que as menores foram para fazenda SF com mesma copa, porta-enxerto e SI. Portanto, outras causas no manejo da cultura ocorreram para estas diferenças nas taxas da ET que não foram provenientes nem dos tipos de porta-enxertos nem dos sistemas de irrigação.

Tomando-se $\mathrm{ET}_{\mathrm{r}}$ como indicativo de umidade do solo, as condições mais secas e mais úmidas na zona das raízes do limoeiro coincidiram respectivamente com os menores e maiores valores da ET, ocorrendo nas mesmas fazendas,

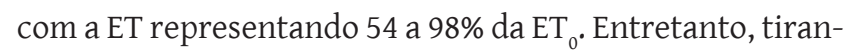
do a média para todos as situações, as taxas evapotranspiratórias com o uso do porta-enxerto Fly Dragon foram 87\% daquelas para o caso de uso do Cravo. Embora com o porta-enxerto Fly Dragon tendo apresentado médias da ET inferiores àqueles para Cravo, o primeiro tipo de porta-enxerto foi usado apenas na fazenda Saara (SA), contabilizando 28,11 ha, enquanto que o Cravo foi utilizado em todas as fazendas em 298,9 ha. Portanto torna-se prematuro a conclusão sobre as diferenças de consumo hídrico com relação ao uso dos dois tipos de porta-enxertos, sendo necessário um aumento da amostragem de cultivos com o Fly Dragon para conclusões sobre o efeito do tipo de porta-enxerto utilizado no consumo hídrico do limoeiro irrigado.

Com os dados de produtividade (P) dos limões obtidos nas fazendas produtoras e da ET modelada pelo algoritmo SAFER, foi possível a obtenção da produtividade da água baseada na evapotranspiração $\left(\mathrm{PA}_{\mathrm{ET}}=\mathrm{P} / \mathrm{ET}\right)$, para cada parcela cultivada. Os maiores valores de $\mathrm{PA}_{\mathrm{ET}}$, de 3,5 a 3,9 $\mathrm{kg} \mathrm{m}^{-3}$ coincidiram com os menores valores da $\mathrm{ET}$ e da $\mathrm{ET}_{\mathrm{r}}$, nas fazendas SA e SF, em parcelas envolvendo portas enxertos tanto Cravo como Fly Dragon e sob ambos os tipos de irrigação localizada ( $\mathrm{M}$ e $\mathrm{G}$ ). Com a produtividade de 37,5 a 40,1 tha ${ }^{-1}$ nestas condições, 50 a $60 \%$ acima da média da região de $25 \mathrm{t} \mathrm{ha}^{-1}$, evidencia-se a grande possibilidade de economia de água com irrigação localizada sem redução 
Tabela 3. Valores anuais dos componentes da produtividade da água sob os diferentes porta-enxertos e sistemas de irrigação (SI) por micro aspersão (M), gotejamento (G) e pivô (P), nas fazendas Esperança (ES), Yamada (YA), Santa Fé (SF), Saara (AS), Marazul (MA) e Tropical (TR). Evapotranspiração real (ET); indicador razão de evapotranspiração $\left(\mathrm{ET}_{\mathrm{r}}\right)$; e produtividade da água baseada na $\mathrm{ET}\left(\mathrm{PA}_{\mathrm{ET}}\right)$.

\begin{tabular}{ccccccc} 
Fazenda & $\begin{array}{c}\text { Porta } \\
\text { Enxerto }\end{array}$ & Copa & SI & $\begin{array}{c}\text { ET } \\
(\mathbf{m m})\end{array}$ & $\begin{array}{c}\mathbf{E T}_{\mathbf{r}} \\
(-)\end{array}$ & $\begin{array}{c}\text { PAET }^{-3} \\
\left(\mathbf{k g ~ m}^{-3}\right)\end{array}$ \\
\hline ES & Cravo & IAC5 & M & $1517 \pm 76$ & $0,93 \pm 0,05$ & 1,7 \\
& Cravo & IAC5 & M & $1188 \pm 59$ & $0,73 \pm 0,04$ & 2,6 \\
YA & Citrumelo & Quebra Galho & P & $1255 \pm 40$ & $0,76 \pm 0,02$ & 2,9 \\
& Cravo & Quebra Galho & P & $1195 \pm 57$ & $0,73 \pm 0,03$ & 2,3 \\
& Cravo & IAC5 & M & $954 \pm 161$ & $0,58 \pm 0,04$ & 3,9 \\
SF & Cravo & IAC5 & M & $1172 \pm 154$ & $0,72 \pm 0,09$ & 2,6 \\
& Cravo & IAC5 & G & $1352 \pm 93$ & $0,83 \pm 0,06$ & 1,2 \\
& Cravo & IAC5 & G & $1182 \pm 171$ & $0,72 \pm 0,11$ & 1,2 \\
SA & Cravo & Quebra Galho & G & $971 \pm 128$ & $0,59 \pm 0,08$ & 3,5 \\
& Fly Dragon & IAC5 & G & $1101 \pm 120$ & $0,68 \pm 0,07$ & 3,6 \\
MA & Fly Dragon & IAC5 & M & $1051 \pm 56$ & $0,65 \pm 0,04$ & 1,4 \\
& Cravo & Quebra Galho & M & $1110 \pm 59$ & $0,69 \pm 0,04$ & 2,2 \\
TR & Cravo & IAC5 & M & $1234 \pm 228$ & $0,76 \pm 0,14$ & 2,9 \\
\hline
\end{tabular}

da produção. Os menores valores da $\mathrm{PA} \mathrm{ET}_{\mathrm{ET}}$, de 1,2 e $1,4 \mathrm{~kg}$ $\mathrm{m}^{-3}$ ocorreram nas mesmas fazendas dos máximos valores e envolvendo ambos os sistemas de irrigação e porta-enxertos.

Como ocorreram baixos e altos valores da $\mathrm{PA}_{\mathrm{ET}}$ tanto com os sistemas $\mathrm{M}$ e $\mathrm{G}$ como e em similares porta-enxertos, não se pôde concluir o efeito destas diferenças de manejo na $\mathrm{PA}_{\mathrm{ET}}$ com irrigação localizada. No caso do sistema de irrigação com pivô (P), os valores foram intermediários, em torno de 2,6 $\mathrm{kg} \mathrm{m}^{-3}$ na fazenda YA, sendo ligeiramente superior com o emprego do porta-enxerto Citrumelo. Considerando-se todas as situações apresentadas na Tabela 3, a $\mathrm{PA}_{\mathrm{ET}}$ média foi de $2,4 \mathrm{~kg} \mathrm{~m}^{-3}$, sem diferenciação dos valores com o emprego dos sistemas de irrigação localizada, M e G.

Shatanawi e Suleiman (2007) encontraram valores de $\mathrm{PA}_{\mathrm{ET}}$ para limoeiro na Jordânia, na faixa de 2,3 a 4,2 $\mathrm{kg} \mathrm{m}^{-3}$, variando de acordo com as condições de estresse hídrico. Os valores para irrigação sem déficit foram em torno de 2,2 $\mathrm{kg} \mathrm{m}^{-3}$, muito similares ao do estudo corrente. Os autores observaram aumentos na $\mathrm{PA}_{\mathrm{ET}}$ com algum grau de estresse hídrico estabelecido, dependendo da fase da cultura.

\section{Coeficiente de cultura}

Considerando-se todas as situações apresentadas na Tabela 3, os valores máximos resultantes das médias da $\mathrm{ET}_{\mathrm{r}}$ somados aos desvios padrões durante o ano de 2015, foram considerados como os coeficientes de cultura $\left(\mathrm{K}_{\mathrm{c}}\right)$ para os casos de uso dos sistemas de irrigação por micro aspersão (M), gotejamento (G) e pivô (P), da cultura do limoeiro no
Norte de Minas Gerais, Sudeste do Brasil. Estes valores, apresentados na Figura 5 podem ser posteriormente recomendados na região para o manejo racional da irrigação da cultura através da disponibilidade dos dados da estação agrometeorológica recentemente instalada, com a meta de melhoria da produtividade da água da cultura.

Como não se detectaram diferenças significativas entre os sistemas de irrigação localizada $\mathrm{M} \mathrm{e} \mathrm{G}$, os valores mensais de $\mathrm{K}_{\mathrm{c}}$ podem ser considerados como as médias entre eles, os quais apresentaram um pico de 1,12 no mês de junho e um mínimo de 0,70 no mês de novembro. Considerando um único pivô analisado na região de estudo, o $\mathrm{K}_{c}$ máximo para este sistema foi de 1,03 em julho e o mínimo de 0,69 em dezembro. Nota-se os valores mais baixos do sistema P com relação aos sistemas de irrigação localizada $M$ e $G$ na época chuvosa, enquanto que no período seco os valores no sistema $P$ foram superiores. Uma explicação plausível para estes valores mais elevados nas condições climaticamente mais secas seria uma maior evaporação da água proveniente dos aspersores do pivô antes de chegar na superfície cultivada.

Consoli e Papa (2013) encontraram faixas de $K_{c}$ entre 0,20 a 1,10, para laranjeira irrigada por gotejamento nas condições semiáridas do Mediterrâneo, através de medições de campo. Embora apresentando valores inferiores, provavelmente devidos às diferentes condições climáticas, os valores máximos foram bem próximos daqueles para limoeiro no atual estudo. Entretanto, Junior et al. (2008) reportaram valores de $\mathrm{K}_{\mathrm{c}}$ ligeiramente superiores ao do estu- 


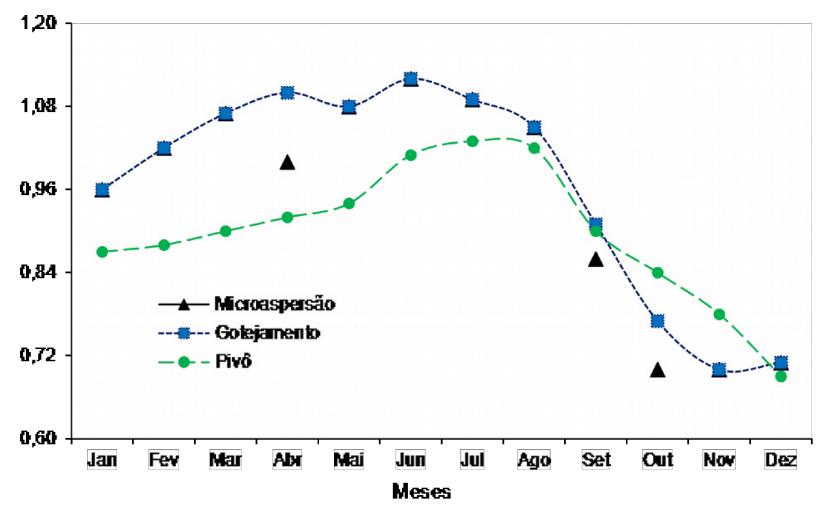

Figura 4. Valores de coeficiente de cultura $\left(\mathrm{K}_{c}\right)$ para o limoeiro irrigado no Norte de Minas Gerais, Sudeste do Brasil, obtidos para os sistemas de irrigação por micro aspersão $(M)$, gotejamento $(G)$ e por pivô (P) no ano de 2015.

do corrente, entre 0,82 e 1,18 $\mathrm{mm} \mathrm{dia}^{-1}$, porém com média de 0,98 bem próxima, resultantes de medições lisimétricas em limoeiro irrigado por gotejamento e porta-enxerto citrumelo, no Sudeste do Brasil.

Considerando os valores médios de todas as situações expostas na Tabela 3 e representadas na Figura 4, bem como as fases apresentadas na Tabela 1, tem-se valores de $\mathrm{K}_{c}$ de 1,01 e 0,97 para a primeira (março a abril) e a segunda (agosto a setembro) floradas (F), respectivamente. Com relação as fases de crescimento dos frutos (CF) tem-se os valores de $\mathrm{K}_{\mathrm{c}}$ de 1,04 de abril a junho e de 0,80 de setembro a novembro. Nos picos de colheita (PC) os valores médios de $\mathrm{K}_{\mathrm{c}}$ foram de $0,88,1,06$ e de 0,72 para os períodos respectivos de janeiro a fevereiro, de junho a agosto e de novembro a dezembro.

No manejo de irrigação atual das fazendas produtoras de limão credenciadas na ASLIM, os valores de $K_{c}$ adotados são de 1,10 e 1,20 nas fases de mais ativas de florescimento e crescimento dos frutos e de 1,00 e 1,10 nas outras situações durante o ano, quando usados os porta-enxertos Cravo e Fly Dragon, respectivamente. Os resultados do atual estudo indicam que valores inferiores de $\mathrm{K}_{\mathrm{c}}$ podem ser usados em conjunto com os dados de $\mathrm{ET}_{0}$ obtidos da estação agrometeorológica, proporcionando economia de água com manutenção da produtividade. Esta implementação pode contribuir ainda para minimização dos possíveis conflitos com outros setores em situação de escassez hídrica nas condições semiáridas da região.

\section{Conclusões}

O uso conjunto de imagens Landsat 8 e dados climáticos permitiu a quantificação e análises dos componentes da produtividade da água em nível de parcelas de irrigação do limoeiro irrigado por diferentes sistemas de irrigação e porta-enxertos durante o ano de 2015, no município de
Matias Cardoso, estado do Minas Gerais, Sudeste do Brasil. As variações espaciais e temporais do indicador de umidade razão da evapotranspiração obtidos pelo algoritmo SAFER demonstrou a forte sensibilidade de detecção das condições hídricas e de vegetação na região. Foi demonstrado que os valores da evapotranspiração (ET), produtividade da água baseada na ET $\left(\mathrm{PA}_{\mathrm{ET}}\right)$ e do coeficiente de cultura $\left(\mathrm{K}_{\mathrm{c}}\right)$ na cultura podem ser estimados através de medições das radiações nas faixas do visível e infravermelho próximo, tendo-se a disponibilidade de dados climáticos de uma estação agrometeorológica próxima à cultura. Os resultados indicaram a possibilidade de uso de valores inferiores de $K_{c}$, com relação aos que vem sendo atualmente usados no manejo de irrigação, o que proporcionaria economia de água com manutenção da produtividade de limões.

\section{Referências}

ALLEN R. G. et al. Crop Evapotranspiration: Guidelines for Computing Crop Water Requirements; Food and Agriculture Organization of the United Nations: Rome, Italy, 1998, 300p.

CONSOLI, S.; PAPA, R. Corrected surface energy balance to measure and model the evapotranspiration of irrigated orange orchards in semi-arid Mediterranean conditions. Irrigation Science, v. 31, p. 1159-1171, 2013.

COSTA, D. S. M.; RUAS, K. F.; PEREIRA, A. M. As potencialidades da região semiárida do Norte de Minas Gerais: Análise do Centro de Estudos de Convivência com o Semiárido. In: ENCONTRO NACIONAL DOS GEÓGRAFOS, 16, 2010, Porto Alegre. Anais... Lavras: AGB, 2010. p. 1-10.

GARCIA-TEJERO, I. et al. Response of citrus trees to deficit irrigation during different phenological periods in relation to yield, fruit quality, and water productivity. Agricultural Water Management. v. 97, p. 689-699, 2010.

GERMANÁ, C.; SARDO, V. Determination when to initiate irrigation of orange trees. Acta Horticulturae, v. 664, p. 591-597, 2004.

JUNIOR, C. R. A. B. et al. Coeficiente de cultura da lima-ácida Tahiti no outono-inverno determinado por lisimetria de pesagem em PiracicabaSP. Engenharia Agrícola, v. 28, n. 4, p. 691-698.

KAMBLE, B.; KILIC A.; HUBARD, K. Estimating crop coefficients using remote sensing-based vegetation index. Remote Sensing, v. 5, p. 15881602,2013

LU, N. et al. Evapotranspiration and soil water relationships in a range of disturbed and undisturbed ecosystems in the semi-arid Inner Mongolia, China. Journal of Plant Ecolology, v. 4, p. 49-60, 2011.

PEDROSO, F. K. J. V. et al. Drought tolerance in citrus trees is enhanced by rootstock dependente changes in root growth and carbohydrate availability. Environmental and Experimental Botany, v. 101, p. 26-35, 2014.

ROBLES, J. M.; BOTÍA, P.; PÉREZ-PÉREZ, J. G. Sour Orange rootstock increases water productivity in deficit irrigated 'Viena' lemon trees compared with Citrus macrophylla. Agricultural Water Mangement, v. 186, p. 98-107, 2017.

SHATANAWI, M.; SULEIMAN, A. A. Lemon evapotranspiration and yield under water déficit in Jordan valley. In: LAMADDALENA, N. et al. (Ed.). Water saving in Mediterranean agriculture and future research needs. CIHEAM: Bari, 2007. p. 63-71.

TEIXEIRA, A. H. de C.; LEIVAS, J. F.; SILVA, G. B. Options for using Landsat and RapidEye satellite images aiming the water productivity assessments in mixed agro-ecosystems. Proceedings of SPIE, v. 9998, p. 99980A-1-99980A-11, 2016. 
TEIXEIRA, A. H. de C; LEIVAS, J. F. Determinação da produtividade da água com imagens Landsat 8 na região semiárida do Brasil. Revista Conexões-Ciência e Tecnologia, v. 10, p. 1-12, 2017.

ZHANG, F. et al. Evapotranspiration and crop coefficient for a temperate desert steppe ecosystem using eddy covariance in Inner Mongolia,

China. Hydrological Processes, v. 26, p. 379-386, 2012.
YANG, L. S.; YANO, T. M. A.; LI, S. Evapotranspiration of orange trees in greenhouse lysemeters. Irrigation Science, v. 21, p. 145-149, 2003.

\section{REFERENCIAÇÃO}

TEIXEIRA, A. H. de C.; REIS, J. B. R. S; LEIVAS, J. F.; BAYMA-SILVA, G.; STRUIVING, T. B. Componentes da produtividade da água modelados por sensoriamento remoto em limoeiros irrigados de Minas Gerais. Agrometeoros, Passo Fundo, v.25, n.1, p.237-247, 2017.

Declaração: os trabalhos estão sendo publicados nesse número de AGROMETEOROS (v.25, n.1, ago 2017) conforme foram aceitos pelo XX Congresso Brasileiro de Agrometeorologia, realizado de 14 a 18 de agosto de 2017, em Juazeiro, BA e Petrolina, PE, sem revisão editorial adicional da revista. 


\title{
Water productivity components modelled by remote sensing on irrigated lemon crops of Minas Gerais State, Brazil
}

\author{
Antônio Heriberto de Castro Teixeira ${ }^{1(*)}$, João Batista Ribeiro da Silva Reis ${ }^{2}$, Janice Freitas Leivas ${ }^{3}$, \\ Gustavo Bayma Siqueira da Silva ${ }^{4}$, Tiago Barbosa Struiving ${ }^{5}$ \\ ${ }^{1}$ Pesquisador, Embrapa Monitoramento por Satélite, Campinas, São Paulo, heriberto.teixeira@embrapa.br \\ ${ }^{2}$ Pesquisador, Empresa de Pesquisa Agropecuária de Minas Gerais, Nova Porteirinha, Minas Gerais, jbrsreis@epamig.br \\ ${ }^{3}$ Pesquisadora, Embrapa Monitoramento por Satélite, Campinas, São Paulo, janice.leivas@embrapa.br \\ ${ }^{4}$ Analista, Embrapa Monitoramento por Satélite, Campinas, São Paulo, gustavo.bayma@embrapa.br \\ ${ }^{5}$ Agrônomo, Associação dos Produtores de Limão, Matias Cardoso, Minas Gerais, struiving@gmail.com \\ ${ }^{(*)}$ Corresponding author
}

\section{ARTICLE INFO}

Article history:

Received 16 June 2017

Accepted 10 August 2017

Index terms:

SAFER

irrigation management

Citrus limon L.

\section{ABSTRACT}

Aiming subsidizing the rational irrigation management for irrigated lemon crops, under different irrigation system and rootstocks, Landsat 8 (L8) images and weather data were used throughout the SAFER algorithm during the year 2015 for modelling the water productivity components in the Matias Cardoso County, Minas Gerais state, Southeast Brazil. For the micro sprinkler and drip irrigation systems, the periods with highest water consumptions, with an actual evapotranspiration (ET) above $4.0 \mathrm{~mm}^{\text {day }}{ }^{-1}$ were from the second half of February to the first half of March. For the pivot system, the maximums, with ET above $4.5 \mathrm{~mm}$ day $^{-1}$ happened from the end of October to the start of November. Considering all irrigation systems and rootstocks used, the water productivity based on ET ( $\left.\mathrm{WP}_{\mathrm{ET}}\right)$ averaged $2.4 \mathrm{~kg}$ $\mathrm{m}^{-3}$. The crop coefficient $\left(\mathrm{K}_{\mathrm{c}}\right)$ ranged according to the considered crop stages in the study region values, being of 1.01 e 0.97 for the two bloom phases; 1.04 and 0.80 for the phases of fruit growth; and of $0.88,1.06$ and 0.72 for the three phases of peak harvests. The results indicated water saving in possibilities the irrigation management while maintaining the crop yield levels. 